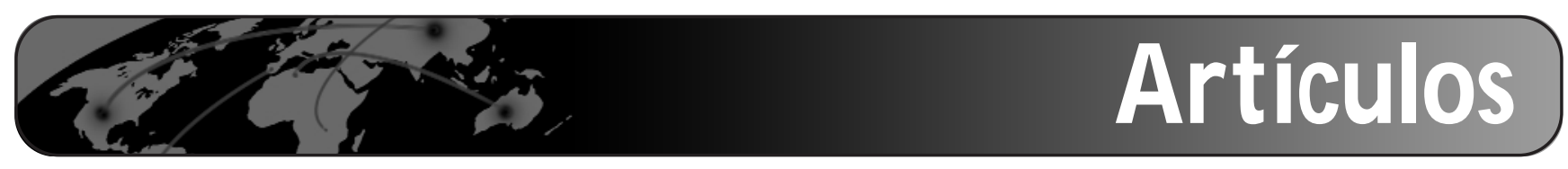

\title{
Revistas electrónicas en las webs de las bibliotecas universitarias españolas
}

\author{
Por José Luis Herrera Morillas
}

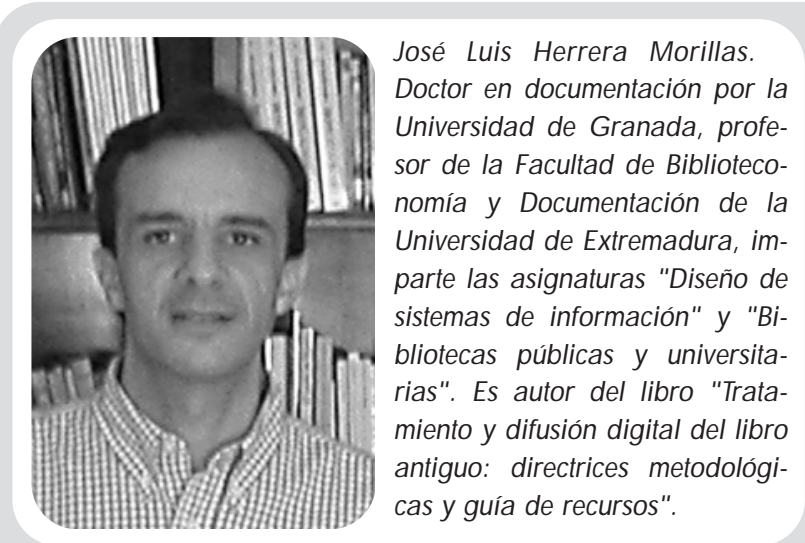

\begin{abstract}
Resumen: La presencia y el uso de las revistas electrónicas se están incrementando en las bibliotecas como una de las manifestaciones del impacto de las nuevas tecnologías. En este trabajo se analiza la información y el acceso en relación con las revistas electrónicas de 57 bibliotecas universitarias españolas disponibles a través de sus páginas web.

Palabras clave: Revistas electrónicas, Revistas científicas, Acceso a revistas electrónicas, Gestión bibliotecaria, Bibliotecas universitarias españolas.

\section{Title: Electronic journals in the web sites of the Spanish university libraries}

\begin{abstract}
The presence and the use of the electronic journals are increasing in the libraries as a manifestation of the impact of the new technologies. In this work we analize the information and the access related to the electronic journals of 57 Spanish university libraries, available through their web sites.
\end{abstract}

Keywords: Electronic journals, Scientific journals, Electronic access, Library management, Spanish university libraries.

Herrera Morillas, José Luis. “Revistas electrónicas en las webs de las bibliotecas universitarias españolas”. En: El profesional de la información, 2004, septiembre-octubre, v. 13, n. 5, pp. 328-337.

\section{Introduc ción}

Hoy en día las bibliotecas son objeto del impacto de la cultura digital y se tiende a la biblioteca híbrida y/o a la biblioteca digital. En el primer caso se habla de aquella que reúne sistemas y servicios integrados en los dos entornos electrónico e impreso; en el segundo, la mayoría de los expertos la conciben como repertorios de objetos digitales, más o menos organizados, que sirven a una comunidad de usuarios definida, respetan los derechos de autor y los gestionan, disponiendo de mecanismos de preservación y conservación apropiados.

El acceso en línea a las revistas electrónicas ofrecido por las bibliotecas universitarias (objeto de este trabajo) es una manifestación concreta de esta nueva concepción de la biblioteca.

La revista digital se ha definido como aquella publicación periódica creada mediante medios electrónicos y que para ser consultada requiere de un hardware y un software específicos. Es responsabilidad de una institución científica o académica, que cumple una función avaladora de la calidad de los contenidos, y puede ser la extensión de una originaria en soporte papel o ser de nuevo cuño'.

Para explicar y justificar la rápida difusión y aceptación de esta nueva modalidad de revista se ha fijado una serie de ventajas ${ }^{2}$, por ejemplo:

—Reducción de costes frente a la impresión.

—Rapidez en maquetación y composición.

—Distribución fácil y sin apenas coste.

-Incorporación de nuevos soportes documentales.

—Posibilidad de actualización permanente de los textos.

—Interactividad autor/lector.

-Facilidad de acceso desde cualquier parte, sin acudir a la biblioteca, y a cualquier hora.

- La posibilidad de eliminar duplicados de revistas en las bibliotecas de una misma red.

—El acceso simultáneo de varios usuarios. 


\title{
Versión online de EPI
}

Existe una versión electrónica de El profesional de la información, de uso gratuito para la mayoría de los suscriptores (empresas, organismos, instituciones), que pueden acceder a través de internet a los textos completos y materiales gráficos publicados en la revista.

\author{
Más información en: \\ http://taylorandfrancis.metapress.com/link.asp?id=105302
}

Dentro de los trabajos dedicados al estudio de las revistas electrónicas, tanto fuera como dentro de nuestro país, los que más abundan son los centrados en la adquisición, gestión y catalogación de estos materiales $^{3}$. Los más cercanos al trabajo que presentamos, por estar dedicados a su acceso y presentación a través de internet, son los siguientes:

- Jordan y Kisly (2002) examinaron durante agosto y septiembre de 2001 una amplia muestra de bibliotecas de todo el mundo para comprobar el acceso y uso de las revistas electrónicas. Para ello diseñaron 37 preguntas que fueron contestadas por 220 bibliotecas (públicas, universitarias y especializadas) de Norteamérica, Europa, Asia y África. El objetivo central era obtener información cuantitativa sobre cómo se proporcionaba y manejaba el acceso a todo tipo de publicaciones electrónicas seriadas. El trabajo destacó la gran diversidad de procedimientos y la carencia de estándares comunes.

\section{«Dentro de los trabajos dedica- dos al estudio de las revistas electrónicas, los que más abundan son los centrados en la adquisición, gestión y catalo- gación de estos materiales»}

- Rich y Rabine (1999) realizaron un estudio sobre la forma por la que las bibliotecas universitarias norteamericanas proporcionan el acceso a las publicaciones periódicas. Es destacable la metodología empleada, siendo la fuente de estudio las propias páginas web, elaborándose un cuestionario muy completo para analizarlas centrado en aspectos como los tipos de acceso, contenidos etc. Se analizan 114 sitios de universidades.

-En España, sobre bibliotecas universitarias tenemos el trabajo de Olmeda y Catalán (2001) que examinan el contenido de las páginas web con información de revistas electrónicas y/o boletines de sumarios electrónicos de las bibliotecas universitarias madrileñas, si bien se limitan a reflexionar sobre el fenómeno del "outsourcing" como estrategia iniciada para hacer frente a la crisis de distribución de la información científico técnica. Sobre la presentación y acceso a través de internet existen dos trabajos de ámbito catalán, el de García (1997) y el de Estivill, Argudo y Miralpeix (2002); este último en concreto es el más cercano y el que ha inspirado nuestra investigación: en una primera parte se analizan las páginas web mediante el diseño de un cuestionario, enfocado fundamentalmente al estudio del tipo de acceso, categorías de materiales incluidos en la colección, criterios de selección y facilidad de acceso a la colección. La segunda parte aborda el paso a las revistas electrónicas desde el catálogo: las políticas de inclusión de estos materiales y el tratamiento catalográfico.

Conscientes de que las revistas científicas impresas han sido el medio del que tradicionalmente se ha servido la comunidad académica e investigadora para difundir los resultados de sus trabajos profesionales y de investigación, y de que estamos asistiendo a un cambio revolucionario en las condiciones de uso de las mismas, dedicamos este estudio a las revistas científicas digitales que presentan las principales bibliotecas universitarias españolas.

\section{Objetivos y metodología}

El objetivo general de este trabajo ha consistido en comprobar el estado actual de las revistas electrónicas disponibles en las páginas web de las bibliotecas universitarias españolas ${ }^{4}$. Para ello hemos establecido unos objetivos concretos, basados en conocer y estudiar la siguiente información:

—Número aproximado de títulos disponibles.

-Tipo de acceso a las revistas (títulos, materias, editor/proveedor, otros).

-Clasificación empleada en el acceso por materias. 


\begin{tabular}{|c|c|c|c|c|c|c|}
\hline $\begin{array}{c}\text { Blblloteca } \\
\text { universitaria }\end{array}$ & $\begin{array}{l}\text { Nümero de } \\
\text { títulos }\end{array}$ & Clasificación materias & $\begin{array}{c}\text { Acceso a revlstas en } \\
\text { prueba }\end{array}$ & $\begin{array}{c}\text { Acceso a revistas sin texto } \\
\text { completo }\end{array}$ & Uso de gestor base de datos & $\begin{array}{c}\text { Acceso a través del catälogo } \\
\text { general }\end{array}$ \\
\hline \multicolumn{7}{|c|}{$\begin{array}{l}\text { pruepa } \\
\text { Andalucia }\end{array}$} \\
\hline Almería & 4.250 & no & no & si & si & no \\
\hline Cádiz & 16.897 & no & si & si & si & si \\
\hline Córdoba & 4.500 & no & si (en novedades) & si & no & si (en fase de realización) \\
\hline Granada & 6.300 & si ( 30 materias generales) & no & no & no & si \\
\hline Huelva & 4.445 & si ( 26 materias generales) & no & no & si & no \\
\hline Jaén & 4.087 & $\begin{array}{c}\text { si (15 materias generales de lo5 } \\
\text { distribuidores) }\end{array}$ & no & & si & si \\
\hline Mälaga & 5.000 & no (palabras clave en catálogo) & $5 i$ & no & & si \\
\hline Pablo Olavide & 5.000 & no (palabras clave en catálogo) & $\begin{array}{c}\text { si (en sección diferenciada } \\
\text { "Revistas-e en periodo de } \\
\text { prueba") }\end{array}$ & no & no & si \\
\hline Sevila & 10.174 & $\begin{array}{c}\text { si (propia adaptada a las titulaciones } \\
\text { impartidas, } 75 \text { materias generales y } \\
\text { especificas) }\end{array}$ & si & si & si & si \\
\hline \multicolumn{7}{|c|}{ - } \\
\hline Zaragoza & 5.274 & no (palabras clave en catàlogo) & $5 \mathrm{i}$ & no & $5 \mathrm{i}$ & no (en proyecto) \\
\hline \multicolumn{7}{|c|}{ Asturlas } \\
\hline Oviedo & 3.940 & no & si & si & no & si (en fase de realización) \\
\hline \multicolumn{7}{|c|}{ Baleares } \\
\hline Islas Baleares & 9.000 & $\begin{array}{c}\text { si (Lista de encabezamientos de materia } \\
\text { Biblioteca de Catalunva) }\end{array}$ & no & no & si & si (en fase de realización) \\
\hline \multicolumn{7}{|c|}{ 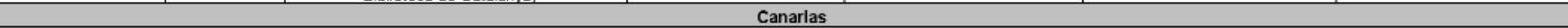 } \\
\hline La Laguna & 2.164 & si ( 28 materias generales) & no & no (en proyecto) & si & si \\
\hline Las Palmas & 3.670 & si (99 materias generales y especificas) & no & no & si & si \\
\hline \multicolumn{7}{|c|}{ Cantabria } \\
\hline Cantabria & 3.000 & $\begin{array}{l}\text { si (propia adaptada a las titulaciones } \\
\text { impartidas. } 29 \text { materias) }\end{array}$ & si & no & si & si[i] \\
\hline \multicolumn{7}{|c|}{ Castila y León } \\
\hline Burgos & 3.793 & si (21 materias generales) & si & $\begin{array}{l}\text { a sumarios electrónicos } \\
\text { (recurso independiente) }\end{array}$ & no & no (en proyecto) \\
\hline León & 7.000 & si (CDU) & no & $5 i$ & si & si (en fase de realización) \\
\hline $\begin{array}{l}\text { Pontificia de } \\
\text { Salamanca }\end{array}$ & $100[i]$ & si (10 materias generales) & no[iii] & si[iv] & no (en proyecto) & no (en proyecto) \\
\hline Salamanca & 8.000 & no (en proyecto) & no & $\begin{array}{l}\text { a sumarios electrónicos } \\
\text { (recurso independiente) }\end{array}$ & no (en proyecto) & si (en fase de realización) \\
\hline Valladolid & 2.460 & no & no & no & no & no \\
\hline \multicolumn{7}{|c|}{ Castllla-La Mancha } \\
\hline Castilla-La Mancha & 4.574 & si ( 26 materias generales) & no & si & si & si \\
\hline & & & Cataluña & & & \\
\hline Autónoma & 5.934 & si (CDU) & $\begin{array}{c}\text { si (en secciòn } \\
\text { diferenciada como } \\
\text { novedad) }\end{array}$ & $\begin{array}{l}\text { a sumarios electrónicos } \\
\text { (recurso independiente) }\end{array}$ & no & si \\
\hline Barcelona & 7.100 & si ( 33 materias generales) & no & no & sا & si \\
\hline Girona & 5.800 & no & si & si & si & si \\
\hline Lleida & 4.990 & si (CDU, abreviada) & no & $\begin{array}{l}\text { a sumarios electrónicos del } \\
\text { CBUC }\end{array}$ & si & si \\
\hline Oberta & 5.650 & si (202 materias generales y especificas) & no & si & si & no \\
\hline Politécnica & 6.000 & si (22 materias generales) & no & si & si & si \\
\hline Pompeu F. & 5.481 & si (Library of Congress) & no & no & $\mathrm{si}$ & si \\
\hline Rovira iV. & 5.890 & no & no & si & si & si \\
\hline Ramon Llull & & & & & & \\
\hline - Esade & 4.500 & si (Library of Congress) & no & $5 i$ (boletin propio) & si & no \\
\hline - Blanquerna & 600 & si ( 7 materias generales) & - & $5 i$ & -- & si \\
\hline- IOS & 850 & no & - & - & - & - \\
\hline Vic & 3.837 & si (Lemac) & no & no & si & $5 i$ \\
\hline & & & Extremadu & & & \\
\hline Extremadura & 2.050 & no & no & si & no & $\begin{array}{l}\text { sí (sólo las de doble versión: } \\
\text { papel y electrónica) }\end{array}$ \\
\hline & & & Gallcla & & & \\
\hline A Coruña & 1.245 & si (Ulrich's y la de Swets) & no & si & no & si \\
\hline Santiago & 17.000 & no (palabras clave en catálogo) & - & si & si & si \\
\hline Vigo & 1.236 & no & no (en proyecto) & si & no & si \\
\hline & & & La Rloja & & & \\
\hline La Rioja & 1.700 & no & no & si & si & no \\
\hline & & & Madrld & & & \\
\hline Alcalá & 2.500 & no (palabras clave en catálogo) & - & - & si & si \\
\hline Carlos III & 6.089 & si (17 materias generales) & - & - & si & $5 i$ \\
\hline Comillas & -- & 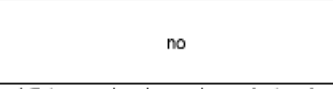 & no & $\begin{array}{l}\text { a sumarios electrónicos } \\
\text { (recurso independiente) }\end{array}$ & no & $\begin{array}{l}\text { no (se indica la existencia y } \\
\text { dirección de la versión } \\
\text { electrónica pero sin incluir } \\
\text { enlace) }\end{array}$ \\
\hline Complutense & 4.000 & $\begin{array}{c}\text { si (istas propias de encabezamientos de } \\
\text { materias) }\end{array}$ & si & si & no (en proyecto) & $5 i$ \\
\hline Europea & 190 & no (palabras clave en catälogo) & si & si & $5 \mathrm{si}$ & si \\
\hline Politécnica & 5.000 & no (palabras clave en catálogo) & no & no & si & si \\
\hline Rey Juan C. & 8.675 & si (34 materias generales) & no & $\begin{array}{l}\text { a sumarios electrónicos } \\
\text { (recurso independiente) }\end{array}$ & si & no \\
\hline UNED & 10.000 & si ( 16 materias generales) & si & si & si & si \\
\hline & & & Murcla & & & \\
\hline Católica S. Antonio & 62 & no & no & no & no & no \\
\hline Murcia & 3.018 & no & si & si & si & $\begin{array}{l}\text { si (solo las de doble versión: } \\
\text { papel y electrónica) }\end{array}$ \\
\hline $\begin{array}{l}\text { Politécnica de } \\
\text { Cartagena }\end{array}$ & 3.000 & no & no & $\begin{array}{c}\text { si (desde plataforma de los } \\
\text { editores) }\end{array}$ & no & si \\
\hline & & & Navarra & & & \\
\hline Navarra & 6.000 & si ( 75 materias generales y especificas) & silv] & no & si & si (en fase de realización) \\
\hline & & & País Vasc & & & \\
\hline Deusto & - & si (12 materias generales) & - & - & - & $5 \mathrm{i}$ \\
\hline Pais Vasco & 3.163 & no (palabras clave en catàlogo) & si & no & no & si \\
\hline & & & Valencia & & & \\
\hline Alicante & 2.724 & no (palabras clave en catálogo) & no & 5i & no (en fase de desarrollo) & $5 \mathrm{i}$ \\
\hline Jaume I & 4.169 & si (de los editores) & no & si & si & si (en fase de realización) \\
\hline Miguel Hernández & 1.700 & no & no & no & no & no \\
\hline $\begin{array}{l}\text { Politécnica de } \\
\text { Valencia }\end{array}$ & 3.726 & si ( 26 materias generales) & no & no & no (en proyecto) & si (en fase de prueba) \\
\hline Valencia & 3.800 & si (13 materias generales) & no & $\begin{array}{l}\text { si a sumarios electrónicos } \\
\text { (recurso independiente) }\end{array}$ & si & no (en proyecto) \\
\hline
\end{tabular}

Figura 1. Cuestionario: preguntas contestadas por las bibliotecas 
- Si se incluye acceso a revistas en prueba.

- Si es posible el acceso a revistas sin texto completo (sumarios y/o resúmenes de artículos).

— Si se utiliza un gestor de bases de datos para su organización.

- Si existe acceso a través del catálogo general de la universidad.

- Si tiene página propia para el acceso a las revistas.

- Si se incorporan enlaces a otras instituciones y/o directorios.

—Si existe información complementaria.

—Grado de visibilidad y accesibilidad. revistas.

- Modo de denominar a este tipo de

—Información asociada a los títulos de las publicaciones.

Para estudiarlas hemos elaborado un cuestionario inspirado en el trabajo de Estivill, Argudo y Miralpeix (2002) sobre presentación y acceso a las revistas electrónicas en las universidades catalanas. Este trabajo nos ha parecido muy completo y el más idóneo respecto a la metodología aplicada de los que hemos localizado referentes a bibliotecas universitarias españolas, aunque de una mayor cobertura en cuanto a los aspectos de estudio en relación al trabajo que presentamos.

Parte del cuestionario lo hemos podido aplicar directamente consultando las páginas en internet y otra parte ha sido contestada por las bibliotecas. Fue enviado en enero de 2004 y hemos recibido las respuestas entre los meses de enero a abril; se ha distribuido y respondido por e-mail y también por teléfono. Los datos obtenidos los presentamos en 4 tablas (figuras 1 a 4$)^{5}$.

En total se han analizado 57 bibliotecas universitarias, que son las que hemos localizado con información sobre revistas electrónicas en la web. Cada uno de los centros bibliotecarios se ha considerado como una unidad, pues,

\begin{tabular}{|l|}
\hline \multicolumn{1}{|c|}{ Andalucía } \\
\hline U. de Almería \\
\hline U. de Cádiz \\
\hline U. de Córdoba \\
\hline
\end{tabular}

normalmente las revistas electrónicas se presentan como un recurso único y común para toda la universidad ${ }^{6}$. Las universidades examinadas, distribuidas por comunidades autónomas, se pueden ver en la tabla 1.

Las preguntas a contestar por las bibliotecas fueron las siguientes ${ }^{7}$ :

—Número aproximado de títulos.

-Utilización de clasificación por materias.

-Revistas en prueba.

- Revistas sin texto completo (sumarios y/o resúmenes de artículos).

-Utilización de un gestor de base de datos para la organización de la revistas.

-Acceso a las revistas a través del catálogo general.

La información obtenida mediante la consulta directa de las páginas web la hemos organizado en tres partes:

-Acceso a las revistas desde la web (si cuenta con página propia, enlaces a otras instituciones o directorios; campos de búsqueda: por títulos, materias, editor/proveedor, etc.).

- Criterios de selección y facilidad de consulta (información complementaria; visibilidad y accesibilidad; denominaciones y acceso indirecto).

-Información asociada a los títulos de las revistas electrónicas (año de inicio; texto completo, resúmenes, etc.; restricciones; consulta con password; descripción sumaria del recurso o materia; y otros).

\section{Análisis de los resultados}

Lo hemos organizado en los siguientes apartados:

U. Católica S.

U. Politécnica de Cartagena

Navarra

U. de Navarra

País Vasco

U. de Deusto

U. del País Vasco

U. de Alicante

U. Jaume I

U. Miquel Hernández

U. Politécnica de Valencia

U. de Valencia

Tabla 1
1. Cuestiones contestadas por las bibliotecas.

a. Número aproximado de títulos:

Hemos podido comprobar (figura 1) que el $\mathrm{n}^{\circ}$ aproximado de títulos de revistas electrónicas disponibles en la mayoría de las bibliotecas supera los varios miles. Así, en 22 centros 


\begin{tabular}{|c|c|c|c|c|c|c|}
\hline $\begin{array}{l}\text { Bbblioteca } \\
\text { universitaria }\end{array}$ & $\begin{array}{l}\text { Páginas } \\
\text { propias }\end{array}$ & $\begin{array}{c}\text { Enlaces a otras instituciones o } \\
\text { directorios }\end{array}$ & Acceso por titulos & $\begin{array}{l}\text { Acceso por } \\
\text { materias }\end{array}$ & $\begin{array}{l}\text { Acceso por editor } \\
\text { proveedor }\end{array}$ & Otros tipos de acceso \\
\hline \multicolumn{7}{|c|}{ Andalucía } \\
\hline Almería & si & - revistas gratuitas en la red & $\begin{array}{l}\text { si (titulos y palabras } \\
\text { clave) }\end{array}$ & no & no & no \\
\hline Cádiz & si & no & $\begin{array}{l}\text { si (titulos y palabras } \\
\text { clave) }\end{array}$ & no & si & no \\
\hline Córdoba & si & $\begin{array}{l}\text { - buscadores, sumarios, abstract, } \\
\text { directorios, editores y distribuidores }\end{array}$ & si & no & si & no \\
\hline Granada & si & $\begin{array}{c}\text { - paquetes de revistas } \\
\text { - directorios }\end{array}$ & si & si & no & no \\
\hline Huelva & $\begin{array}{c}\text { si (con la } \\
\text { hemeroteca) }\end{array}$ & no & $\begin{array}{l}\text { si (titulo, inicial, y palabra } \\
\text { clave) }\end{array}$ & si & si & no \\
\hline Jaén & si & no & si & si & no & no \\
\hline Málaga & si & no & $\begin{array}{l}\text { si (campo del catálogo } \\
\text { general) }\end{array}$ & $\begin{array}{l}\text { si (campo del } \\
\text { catálogo general) }\end{array}$ & si & $\begin{array}{c}\text { - palabra clave } \\
\text { - titulo colección } \\
\text { - clasificicación } \\
\text { - Issn } \\
\text { (campos deatálogo } \\
\text { de publicaciones } \\
\text { electrónicas) }\end{array}$ \\
\hline Pablo Olavide & sí & no & si & \begin{tabular}{|c|} 
si \\
(campo del catàlogo \\
qeneral)
\end{tabular} & si & no \\
\hline Sevilla & si & $\begin{array}{l}\text { - Jake, base de datos sobre } \\
\text { recursos-e } \\
\text { - directorios y abreviaturas }\end{array}$ & si & si & si & no \\
\hline \multicolumn{7}{|c|}{ a } \\
\hline Zaragoza & $\begin{array}{l}\text { si (base de } \\
\text { datos Faro) }\end{array}$ & no & si & si & si & no \\
\hline \multicolumn{7}{|c|}{ Asturias } \\
\hline Oviedo & si & - portales y sumarios & si & no & no & no \\
\hline \multicolumn{7}{|c|}{ Baleares } \\
\hline Islas Baleares & si & no & Isi (titulo y palabra clave) & si & no & no \\
\hline \multicolumn{7}{|c|}{ Canarias } \\
\hline La Laguna & si & no & si & si & no & no \\
\hline Las Palmas & si & no & si & si & si & - \\
\hline \multicolumn{7}{|c|}{ Cantabria } \\
\hline Cantabria & sí & - sumarios, alertas, índices & si & si & si & no \\
\hline \multicolumn{7}{|c|}{ Castilla y León } \\
\hline Burgos & si & - sumarios & si & si & si & no \\
\hline León & sí & \begin{tabular}{|c|} 
- boletines oficiales, prensa, \\
buscadores de revistas \\
- sumarios de revistas de la \\
Asociación Española de Entomologia \\
\end{tabular} & si & & & no \\
\hline $\begin{array}{l}\text { Pontificia de } \\
\text { Salamanca }\end{array}$ & si & - buscadores de articulos & no & si & no & по \\
\hline Salamanca & si & - directorios, sumarios & si & no & no & no \\
\hline Valladolid & si & no & si & no & si & no \\
\hline \multicolumn{7}{|c|}{ Castilla-La Mancha } \\
\hline $\begin{array}{c}\text { Castilla-La } \\
\text { Mancha }\end{array}$ & si & no & si & si & si & no \\
\hline & & & Cataluña & & & \\
\hline Autónoma & si & no & si & si & si & no \\
\hline Barcelona & si & no & $\begin{array}{l}\text { si (palabra clave, catál. } \\
\text { qeneral) }\end{array}$ & no & no & по \\
\hline Girona & si & no & si & no & si & no \\
\hline Lleida & si & $\begin{array}{l}\text { - sumarios CBUC } \\
\text { - accesso a Acrobat }\end{array}$ & si & $\begin{array}{l}\text { si (en fase de } \\
\text { realización) }\end{array}$ & & no \\
\hline Oberta & si & - directorios titulos de revistas & si & si & no & no \\
\hline Politécnica & si & no & si & si & no & - biblioteca \\
\hline Pompeu F. & si & $\begin{array}{l}\text { - sumarios electrónicos } \\
\text { - buscadores y directorios } \\
\text { - editoriales y servicios } \\
\text { - otros recursos }\end{array}$ & si & si & no & по \\
\hline Rovira iV. & si & no & si & no & no & no \\
\hline Ramon Llull. & & & & & & \\
\hline Esade & si & no & si & si & si & Issn \\
\hline $\begin{array}{l}\text { Blanquerna } \\
\text { IQS }\end{array}$ & $\begin{array}{l}\text { si } \\
\text { si }\end{array}$ & $\begin{array}{l}\text { no } \\
\text { no }\end{array}$ & si & $\begin{array}{l}\text { si } \\
\text { no }\end{array}$ & $\begin{array}{l}\text { no } \\
\text { no }\end{array}$ & $\begin{array}{l}\text { no } \\
\text { no }\end{array}$ \\
\hline IQS & si & $\frac{\text { no }}{\text { - sumarios CBUC }}$ & $\begin{array}{l}\text { si } \\
\text { si }\end{array}$ & $\begin{array}{l}\text { no } \\
\text { si }\end{array}$ & $\frac{\text { no }}{\text { si }}$ & no \\
\hline & & - bases de datos revistas & Extremadura & & & \\
\hline Extremadura & si & no & si & no & si & no \\
\hline & & & Galicia & & & \\
\hline A Coruña & si & no & si & si & si & no \\
\hline Santiago & si & - portales & si & $\begin{array}{l}\text { si (campo del } \\
\text { catälogo qeneral) }\end{array}$ & si & - Issn \\
\hline Vigo & si & no & si & no & no & no \\
\hline & & & La Rioja & & & \\
\hline La Rioja & si & no & no & no & si & no \\
\hline & & & Madrid & & & \\
\hline Alcaláa & si & $\begin{array}{c}\text { - servicios de editores y proveedores } \\
\text { acceso a texto completo } \\
\text { - bases de datos texto completo } \\
\text { - sumarios } \\
\end{array}$ & $\begin{array}{c}\text { si (campo del catálogo } \\
\text { general) }\end{array}$ & $\begin{array}{c}\text { si (campo del } \\
\text { catálogo general) }\end{array}$ & $\begin{array}{l}\text { a travès del } \\
\text { catálogo }\end{array}$ & no \\
\hline Carlos III & si & no & si & si & si & no \\
\hline Comilas & si & no & si & no & no & no \\
\hline Complutense & si & no & si & si & si & - colecciones \\
\hline Europea & si & no & si & $\begin{array}{l}\text { si (campo del } \\
\text { catálogo general) }\end{array}$ & & \\
\hline Politécnica & si & no & si & $\begin{array}{c}\text { si (campo del } \\
\text { catálogo general) }\end{array}$ & no & no \\
\hline Rey Juan C. & si & no & si & si & si & no \\
\hline UNED & si & no & si & si & si & no \\
\hline & & & Murcia & & & \\
\hline $\begin{array}{l}\text { Católica S. } \\
\text { Antonio }\end{array}$ & si & no & si & no & no & no \\
\hline Murcia & si & no & si (listado a-z de Ebsco) & no & si & no \\
\hline $\begin{array}{l}\text { Politècnica de } \\
\text { Cartagena }\end{array}$ & si & no & si & no & no & no \\
\hline & & & Navarra & & & \\
\hline Navarra & si & - portales de revistas electrónicas & $\begin{array}{c}\text { si (campo del catálogo } \\
\text { Reviel) }\end{array}$ & $\begin{array}{l}\text { si (campo del } \\
\text { catälogo Reviel) }\end{array}$ & no & nо \\
\hline & & & Pais Vasco & & & \\
\hline Deusto & si & no & si & si & no & no \\
\hline Pais Vasco & si & $\begin{array}{l}\text { - servicios de alertas, sumarios } \\
\text { electronicos }\end{array}$ & $\begin{array}{c}\text { si (campo del catälogo } \\
\text { qeneral) }\end{array}$ & $\begin{array}{c}\text { si (campo del } \\
\text { catálogo qeneral) }\end{array}$ & si & по \\
\hline & & & Valencia & & & \\
\hline Alicante & si & no & no & no & si & no \\
\hline Jaume I & si & no & $\begin{array}{c}\text { si (campo del catàlogo } \\
\text { de revistas) }\end{array}$ & $\begin{array}{c}\text { si (campo del } \\
\text { catálogo de revistas) }\end{array}$ & $\begin{array}{l}\text { si (campo del } \\
\text { catálogo de } \\
\text { revistas) }\end{array}$ & $\begin{array}{l}\text {-ISSN } \\
\text { - proveedor } \\
\text { - fondo }\end{array}$ \\
\hline $\begin{array}{l}\text { Miguel } \\
\text { Hernandez }\end{array}$ & $\begin{array}{c}\text { si (común } \\
\text { para todas las } \\
\text { revistas) }\end{array}$ & $\begin{array}{c}\text { - portales de revistas y otros } \\
\text { servicios }\end{array}$ & si & no & no & no \\
\hline $\begin{array}{c}\text { Politécnica de } \\
\text { Valencia }\end{array}$ & si & no & si & si & si & nо \\
\hline Valencia & & - portales, sumarios, directorios & si & si & no & no \\
\hline
\end{tabular}

Figura 2. Cuestionario: acceso a las revistas desde páginas web
$(38,5 \%)$ tienen más de 5.000. Las universidades que más destacan en cifras globales son las de Cádiz, Sevilla, Islas Baleares, Santiago y UNED con valores desde los 10.000 títulos.

b. Clasificación por materias:

31 bibliotecas (54,3\%) presentan algún tipo de clasificación por materias. De ellas, 15 $(26,3 \%)$ han adoptado clasificaciones formadas por materias generales, que oscilan entre las 10 áreas temáticas (Pontificia de Salamanca) y las 34 (Rey Juan Carlos). Otros centros como Navarra, Oberta, Las Palmas o Sevilla han optado por herramientas más desarrolladas, donde las materias generales se completan con otras más específicas. También las hay que han escogido célebres clasificaciones como la $C D U$ (León, Autónoma de Barcelona, Lleida), Library of Congress (Pompeu Fabra, Ramón Llull-Esade), Lista de Encabezamientos de Materia de la Biblioteca de Cataluña (Islas Baleares, Vic); o que utilizan dos o más sistemas ( $A$ Coruña, Alicante). 8 bibliotecas (Málaga, Pablo Olavide, Santiago, Alcalá, Europea, Politécnica de Madrid, País Vasco y Zaragoza) aunque no ofrecen clasificaciones de materias para el acceso desde la web de las revistas, sí lo permiten a través del catá- 
logo general o de la base de datos específica para las revistas.

c. Revistas en prueba:

Se incluyen en 17 bibliotecas $(29,8 \%)$; en ocasiones las presentan aparte como novedad (Córdoba, Pablo Olavide, Autónoma de Barcelona).

d. Revistas sin texto completo:

32 bibliotecas $(56,1 \%)$ sitúan junto a las de texto completo revistas con sólo sumarios y resúmenes; en $8(14 \%)$ los sumarios electrónicos se muestran como sección independiente.

e. Uso de gestor de bases de datos:

Han sido los propios centros los que nos han informado sobre si utilizan gestores de bases de datos para la organización de las revistas en la página web, y han sido en total 33 bibliotecas $(57,8 \%)$. En algunas se trata de bases de datos elaboradas en Access (Huelva, Islas Baleares, Las Palmas, Lleida, Vic), Filemaker (Zaragoza, Navarra), SQL (Sevilla, Girona, Politécnica de Cataluña). Otras soluciones consisten en aplicaciones del propio sistema integrado de bibliotecas ( $\mathrm{La} \mathrm{La}$ guna, Castilla-La Mancha, Politécnica de Madrid, Pompeu Fabra, Carlos III); servidor IRI (Europea de Madrid); CDS/ISIS (Rovira $i$ Virgili); o han contratado los servicios de Ebsco (UNED, Murcia, León). Finalmente 4 bibliotecas han contestado que tienen en proyecto utilizar un gestor de base de datos (Salamanca, Pontificia de Salamanca, Complutense, Politécnica de Valencia).

f. Acceso a las revistas a través del catálogo:

La última cuestión hace mención a la posibilidad de consultar también las revistas a través del catálogo general de la biblioteca: 42 centros $(73,6 \%)$ proporcionan esta posibilidad. De ellos en 8 (14\%) está en fase de realización (Salaman-

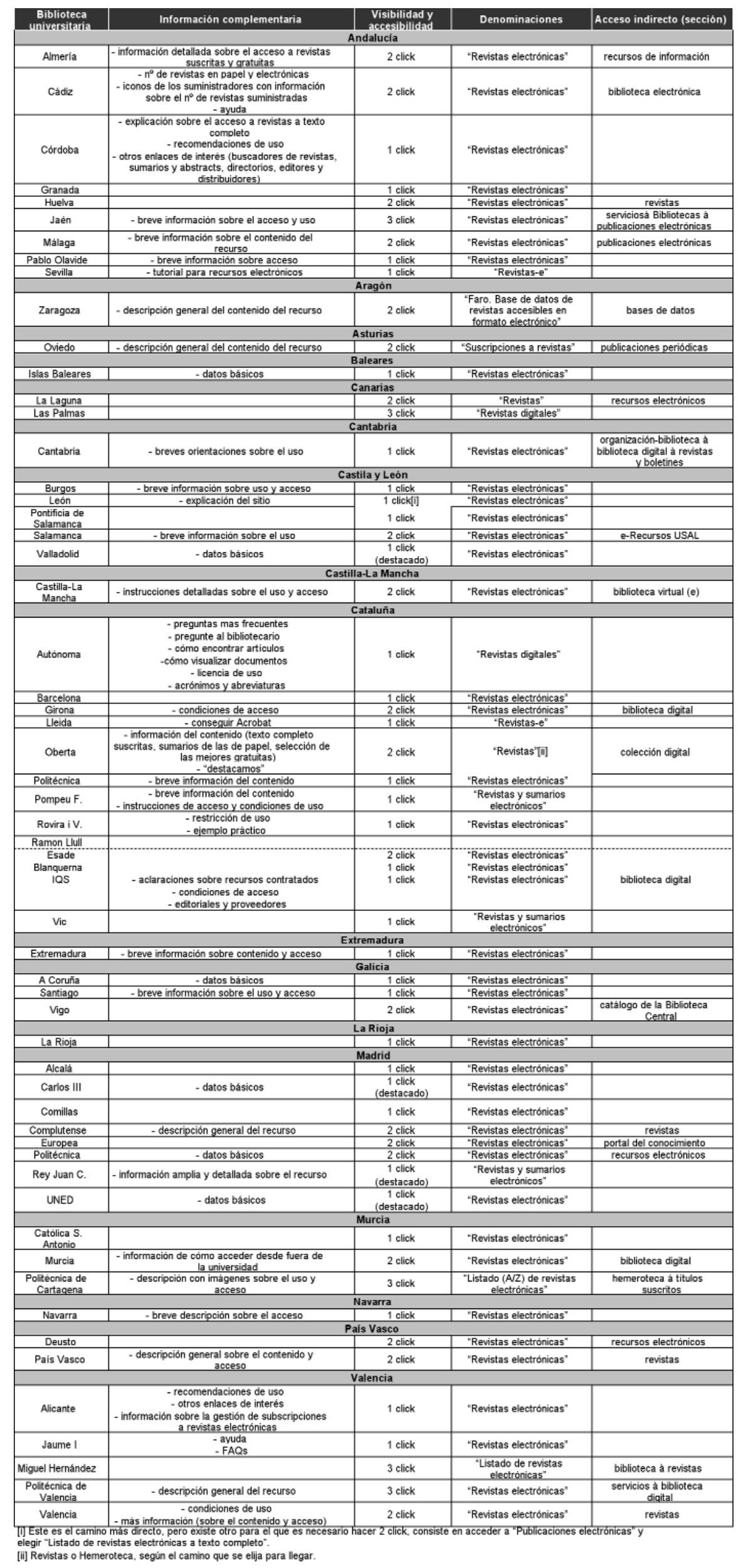

Figura 3. Cuestionario: criterios de selección y facilidad de acceso 


\begin{tabular}{|c|c|c|c|c|c|c|}
\hline $\begin{array}{c}\text { Biblioteca } \\
\text { universitaria }\end{array}$ & Año inicio acceso & $\begin{array}{c}\text { Acceso a texto completo, } \\
\text { resúmenes... }\end{array}$ & $\begin{array}{l}\text { Restricciones de } \\
\text { acceso }\end{array}$ & $\begin{array}{l}\text { Consulta con } \\
\text { password }\end{array}$ & $\begin{array}{c}\text { Descripción sumaria } \\
\text { del recurso o } \\
\text { materia }\end{array}$ & Otros \\
\hline \multicolumn{7}{|c|}{ Andalucia } \\
\hline Almeria & si & si & no & no & no & no \\
\hline Cádiz & $\begin{array}{c}5 i \\
\text { (cobertura } \\
\text { cronológica) }\end{array}$ & si & no & no & no & no \\
\hline Córdoba & $\frac{1}{\text { no }}$ & no & no & no & no & no \\
\hline Granada & $\begin{array}{c}\mathrm{si} \\
\text { (en algunas) }\end{array}$ & no & no & no & no & по \\
\hline Huelva & si & no & no & si & no & $\begin{array}{l}\text { - disponible en papel } \\
\text { - url } \\
\text { - si requiere registro }\end{array}$ \\
\hline Jaén & no & no & no & no & no & no \\
\hline Málaga & no & no & no & no & no & no \\
\hline Pablo Olavide & no & no & no & no & no & \begin{tabular}{|c|} 
- enlace a revistas en periodo de \\
prueba \\
- enlace al registro del catalogo \\
qeneral
\end{tabular} \\
\hline Sevilla & $\begin{array}{c}\text { si } \\
\text { (cobertura } \\
\text { cronolóqica) }\end{array}$ & $\begin{array}{c}\text { si } \\
\text { (texto completo) }\end{array}$ & $\begin{array}{c}\text { si } \\
\text { (restricciones de } \\
\text { uso) }\end{array}$ & no & si & $\begin{array}{c}\text { - acceso remoto } \\
\text { - guia de uso } \\
\text { - } n^{n} \text { de recursos localizados } \\
\end{array}$ \\
\hline \multicolumn{7}{|c|}{ Aragón } \\
\hline Zaragoza & si & $\begin{array}{c}\text { si } \\
\text { (texto completo, sumarios, resúmenes) }\end{array}$ & no & по & \begin{tabular}{|c|}
$\mathbf{s i}$ \\
(información general)
\end{tabular} & no \\
\hline \multicolumn{7}{|c|}{ Asturias } \\
\hline Oviedo & no & no & & no & no & no \\
\hline \multicolumn{7}{|c|}{ Baleares } \\
\hline \multirow{2}{*}{\multicolumn{7}{|c|}{ - "embargement" (en algunas) }} \\
\hline & & & & & & \\
\hline La Laguna & no & no & no & no & no & $\begin{array}{l}\text { - enlace al registro del catálogo } \\
\text { general }\end{array}$ \\
\hline Las Palmas & no & no & no & no & no & $\begin{array}{c}\text { - enlace al distribuidor } \\
\text { - Issn }\end{array}$ \\
\hline \multicolumn{7}{|c|}{ Cantabria } \\
\hline Cantabria & si & no & si & si & no & $\begin{array}{l}\text { - disponible en papel } \\
\text { - formato (html, pdf) } \\
\text { - licencia }\end{array}$ \\
\hline \multicolumn{7}{|c|}{ Castillay León } \\
\hline Burgos & no & no & no & si & no & no \\
\hline León & si & no & no & no & no & $\begin{array}{l}\text { - enlace al distribuidor } \\
\text { - editor } \\
\text { - Issn } \\
\text { - materia } \\
\end{array}$ \\
\hline $\begin{array}{l}\text { Pontificia de } \\
\text { Salamanca }\end{array}$ & $\begin{array}{c}\text { si } \\
\text { (a veces en la } \\
\text { descripción del } \\
\text { recurso) }\end{array}$ & $\begin{array}{c}\text { si } \\
\text { (a veces en la descripción del recurso) }\end{array}$ & no & no & si & - \\
\hline $\begin{array}{l}\text { Salamanca } \\
\text { Valladolid }\end{array}$ & $\begin{array}{l}\text { no } \\
\text { no }\end{array}$ & $\frac{\text { no }}{\text { no }}$ & no & no & no & no \\
\hline \multirow{2}{*}{\multicolumn{7}{|c|}{ Castilla-La Mancha }} \\
\hline & & & & & & \\
\hline Castilia-La Mancha & no & no & no & no & no & - enlace al registro del catälogo \\
\hline & & & Cataluña & & & \\
\hline Autónoma & no & no & no & no & no & no \\
\hline Barcelona & no & no & no & no & no & no \\
\hline Girona & si & no & no & no & no & no \\
\hline Lleida & no & no & no & no & no & $\begin{array}{c}\text { - "novedad" } \\
\end{array}$ \\
\hline Oberta & si & si & si & no & $\begin{array}{l}\text { si } \\
\text { (idioma, autor, } \\
\text { materia }\end{array}$ & $\begin{array}{l}\text { - recurso recomendado } \\
\text { - ámbito geográfico }\end{array}$ \\
\hline Poiltécnica & no & si & no & no & materla & \begin{tabular}{|c|} 
- editor \\
- Issn \\
- suscripción electrónica al sumario \\
(acceso) \\
- enlace al catälogo
\end{tabular} \\
\hline Pompeu F. & & & registros de & tálogo & & \\
\hline Rovira iV. & no & no & no & no & no & no \\
\hline Ramon LluIt. & & & & & & \\
\hline $\begin{array}{l}\text { Eade } \\
\text { Blanquerna }\end{array}$ & si (cobertura) & si & $\begin{array}{l}\text { no } \\
\text { si }\end{array}$ & $\begin{array}{c}\text { no } \\
\text { si }\end{array}$ & $\begin{array}{l}\text { no } \\
\text { no }\end{array}$ & $\begin{array}{l}\text { - editor } \\
\text { no }\end{array}$ \\
\hline $\begin{array}{l}\text { Das } \\
\text { IQS }\end{array}$ & no & -. & no & $\begin{array}{l}\text { no } \\
\text { no }\end{array}$ & no & $\begin{array}{c}\text { no } \\
\text { - editor }\end{array}$ \\
\hline Vic & no & no & no & no & no & no \\
\hline & & & Extremadura & & & \\
\hline Extremadura & no & no & no & no & no & no \\
\hline & & & Galicia & & & \\
\hline A Coruña & no & no & no & no & no & no \\
\hline Santiago & no & no & no & no & no & no \\
\hline Vigo & no & no & no & no & no & no \\
\hline & & & La-Rioja & & & \\
\hline La-Rioja & no & no & no & no & no & no \\
\hline & & & Madrid & & & \\
\hline Alcalá & no & no & no & no & no & no \\
\hline Carlos III & si & no & no & no & no & $\begin{array}{c}\text { - disponible sólo en formato } \\
\text { electrónico } \\
\text { - } 1 \text { copia disponible en internet } \\
\text { - enlace al registro del catálogo } \\
\text { general } \\
\text {-url } \\
\text { - opción guardar }\end{array}$ \\
\hline Comillas & no & no & no & no & no & \begin{tabular}{|c|} 
no \\
\end{tabular} \\
\hline Complutense & si & no & no & no & no & 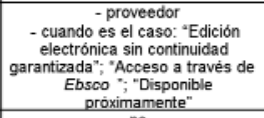 \\
\hline Europea & si & si & no & no & si & no \\
\hline Politécnica & no & no & no & no & no & no \\
\hline Rey Juan C. & si & no & no & no & no & $\begin{array}{l}\text { - editor } \\
\text { - fuente (Universidad o Madroño) }\end{array}$ \\
\hline UNED & $\begin{array}{c}\text { si } \\
\text { (en algunos) }\end{array}$ & no & no & no & no & $\begin{array}{l}\text { - disponible en papel } \\
\text { - proveedor }\end{array}$ \\
\hline & & & Murcia & & & \\
\hline Católica S. Antonio & no & no & no & no & no & no \\
\hline Murcia & & & esos que presenta cas & editor o distribuidor & & \\
\hline $\begin{array}{l}\text { Politécnica de } \\
\text { Cartagena }\end{array}$ & si & no & no & no & no & $\begin{array}{l}\text { - enlace al distribuidor } \\
\text { - editor } \\
\text { - Issn } \\
\text { - materia }\end{array}$ \\
\hline & & & Navarra & & & \\
\hline Navarra & si & no & sí & si & si & no \\
\hline & & & Pais Vasco & & & \\
\hline Deusto & no & no & no & no & no & no \\
\hline Pais Vasco & & & registro del & tálogo & & \\
\hline & & & Valencia & & & \\
\hline Alicante & & acceso a los & portales de los distribui & es y al catálogo bi & iográfico & \\
\hline Jaume I & no & no & no & no & no & no \\
\hline Miguel Hernández & no & no & no & no & no & no \\
\hline $\begin{array}{c}\text { Politécnica de } \\
\text { Valencia }\end{array}$ & si & no & si & no & no & no \\
\hline Valencia[i] & - & - & - & - & -- & -- \\
\hline
\end{tabular}

Figura 4. Cuestionario: información asociada a los títulos de las revistas electrónicas 


\section{Próximos temas especiales}

\section{N oviembre $2004 \quad$ Propiedad intelectual \\ Enero 2005 Posicionamiento en la Web \\ Marzo 2005 Consorcios de bibliotecas}

Los interesados pueden remitir notas, artículos, propuestas, publicidad, comentarios, etc., sobre estos temas a:

\section{epi@ sarenet.es}

ca, Córdoba, Oviedo, Islas Baleares, León, Navarra, Jaume I y Politécnica de Valencia). Sólo 14 (24,5\%) no tienen acceso desde el catálogo, aunque en $4(7 \%)$ existe el proyecto de incluirlo (Zaragoza, Burgos, Pontificia de Salamanca y Valencia) y 1 no contesta.

\section{Acceso a las revistas desde la web.}

En este apartado tratamos las siguientes cuestiones.

a. Páginas propias:

De las 57 bibliotecas analizadas todas cuentan con una página propia para las revistas electrónicas (figura 2), pero con notables diferencias en diseño y contenidos.

b. Enlaces a otras instituciones o directorios:

Dentro de estas páginas 20 bibliotecas (35\%) disponen de enlaces a otras instituciones o recursos relacionados, como directorios de revistas, etc.

c. Tipos de acceso (títulos, materias, editor/proveedor, otros):

Respecto a los campos, en 54 bibliotecas $(94,7 \%)$ es por títulos (en 4 casos se lleva a cabo a través del catálogo general) y sólo 3 (Pontificia de Salamanca, La Rioja y Alicante) no lo proveen. La búsqueda por materias está presente en $39(68,4 \%)$, aunque en 8 casos es a través de un campo del catálogo general. La otra modalidad más difundida es por editor y/o proveedor, que está presente en 30 bibliotecas $(52,6 \%)$. Finalmente se utilizan otras formas: por medio del ISSN (Málaga, Ramon Llull-Esade, Santiago, Jaime I), colección-fondo (Málaga, Complutense, Jaume I) o biblioteca (Politécnica de Cataluña).

3. Criterios de selección y facilidad de consulta.
El tercer grupo de resultados analizados hace referencia a los criterios de selección y facilidad de consulta (figura 3 ).

a. Información complementaria:

40 bibliotecas $(70 \%)$ presentan en la web de revistas electrónicas información de apoyo, como explicación sobre el uso de revistas a texto completo, recomendaciones, información sobre el contenido del recurso, tutoriales, ayuda, etc.

b. Visibilidad y accesibilidad:

Las hemos analizado comprobando el número de clicks para llegar a la sección de las revistas electrónicas. En 32 bibliotecas $(56,1 \%)$ dicha sección es visible directamente desde la página inicial de la biblioteca y por tanto se realiza sólo un click para entrar. En 20 bibliotecas (35\%) 2 clicks y sólo en 5 (8,7\%) (Jaén, Las Palmas, Politécnica de Cartagena, Miguel Hernández y Politécnica de Valencia) hay que realizar 3.

c. Denominaciones:

El tercer contenido analizado en este apartado ha sido la forma de designar a esta sección. La expresión más generalizada es "revistas electrónicas" presente en 44 bibliotecas $(77,1 \%)$. En las 13 restantes $(22,8 \%)$ se han utilizado otras como:

— "Revistas-e" (Sevilla, Lleida).

- "Faro. Base de datos de revistas accesibles en formato electrónico" (Zaragoza).

—“Suscripciones a revistas" (Oviedo).

—“Revistas" (La Laguna, Oberta).

— "Revistas digitales" (Las Palmas, Autónoma de Barcelona). 
- "Revistas y sumarios electrónicos" (Pompeu Fabra, Vic, Rey Juan Carlos).

— "Listado (A/Z) de revistas electrónicas" (Politécnica de Cartagena). nández).

— "Listado de revistas electrónicas" (Miguel Her-

\section{Información asociada a los títulos de las re- vistas electrónicas:}

Finalmente abordamos los datos relativos a la información asociada a los títulos de las revistas (figura 4), algo que sólo incorporan 25 bibliotecas $(43,8 \%)$. En 19 casos $(33,3 \%)$ se indica el año de inicio de disponibilidad de la revista; en 9 bibliotecas $(15,7 \%)$ se informa sobre si se incluye el texto completo, resúmenes, etc.; 6 bibliotecas $(10,5 \%)$ señalan las restricciones de acceso y $5(8,7 \%)$ la necesidad de password para su consulta; por último $6(10,5 \%)$ realizan una breve descripción del recurso junto al título.

\section{«En total se han analizado 57 bibliotecas universitarias, que son las que hemos localizado con información sobre revistas electrónicas en la web»}

Estos son los datos más frecuentes que se presentan asociados a los títulos, pero también hemos localizado otro tipo de información en 18 bibliotecas (31,5\%), para indicar que la revista está disponible en papel, el ISSN, el ámbito geográfico o la materia; para precisar que es un recurso recomendado; o también para acceder mediante un enlace al registro del catálogo general de la biblioteca, a la guía de uso, al distribuidor, etc.

\section{Conclusiones}

-Número de títulos disponibles: las revistas electrónicas, como recurso ofrecido por nuestras bibliotecas universitarias, es ya una realidad muy extendida. Sin embargo existen notables diferencias en cuanto a la cantidad ofrecida (desde las que tienen 65 o 100 títulos, que se corresponden normalmente con las suscripciones en papel, hasta las que superan los 16.000) y sobre todo, en cuanto a las características del servicio a través de las webs. Esta variedad nos hace pensar que estamos todavía en una fase de experimentación en cuanto al diseño del servicio y por tanto ante previsibles cambios y perfeccionamientos.

—El acceso a las revistas: el análisis detenido de cada uno de estos servicios nos ha permitido comprobar cómo en el acceso a las revistas electrónicas la tendencia más innovadora es optar por uno integrado en el catálogo y mediante vías alternativas desde la web de la biblioteca. A través del catálogo porque cada vez más se diseña para contener la totalidad de la colección (tanto los soportes tangibles como los documentos electrónicos) y se tiende a configurar como una única base de datos. A través de vías alternativas desde la web de la biblioteca a partir de páginas o secciones diseñadas ad hoc, para proporcionar como mínimo listados de las revistas por títulos y completadas según los casos por otros tipos de accesos (materias, distribuidores, etc.) y enlaces a otras instituciones y recursos relacionados. Este modelo es, a nuestro entender, el que deben conseguir las bibliotecas que todavía cuentan con un servicio muy básico, por ejemplo cuando sólo ofrecen listados de títulos como enlaces a la revistas o simplemente el acceso directo a los distribuidores-proveedores.

\section{«Estamos todavía en una fase de experimentación en cuanto al diseño del servicio y por tan- to ante previsibles cambios y perfeccionamientos»}

Estos datos confirman la evolución descrita por otros autores: "en un primer momento las bibliotecas han optado por la elaboración de páginas web que incluyen listas de títulos de revistas; posteriormente cuando las colecciones han ido creciendo se han desarrollado diversas modalidades de bases de datos web. De manera progresiva estos procedimientos se han completado con el acceso por medio del catálogo"".

Establecemos, pues, 4 modelos en relación a la presentación y acceso de las revistas electrónicas:

1. Página web con listado de revistas (y demás información según los casos): 12 bibliotecas, $21 \%$.

2. Acceso exclusivo a las revistas a través del catálogo general de la biblioteca: 2 centros (Málaga y Alcalá), 3,5\%.

3. Modelo mixto (abarca los modelos 1 y 2): presentan web con listado y también acceso a través del catálogo general: 40 bibliotecas, $70 \%$.

4. Base de datos exclusiva para las revistas: 1 biblioteca (Zaragoza), 1,7\%.

-Enlaces a otras instituciones y/o directorios: los más generalizados son uno o varios de los siguientes: sumarios, buscadores y directorios. En algunos casos también hay bibliotecas que incorporan: revistas gratuitas, base de datos sobre recursos electrónicos, portales, prensa y boletines oficiales.

-Información complementaria: el tipo de información que incluyen estas páginas se centra fundamentalmente en explicaciones sobre el acceso y uso, 
seguida de datos sobre el contenido. Otros aspectos a los que se le dedica atención, aunque en menor medida, son: número de revistas, ayuda, preguntas más frecuentes, suministradores, cómo encontrar artículos, cómo visualizar documentos, licencia de uso, acrónimos y abreviaturas.

- Grado de visibilidad y accesibilidad: los datos sobre la visibilidad —en 52 bibliotecas $(91,2 \%)$ sólo es necesario 1 o 2 click para acceder a las revistas electrónicas desde la página principal del centro- nos indican que hay una clara intención de destacar este servicio, pues no cabe duda que es muy apropiado para subrayar la nueva imagen de la biblioteca en el entorno digital.

- Modo de denominar a este tipo de revistas: la forma mayoritaria, con notable diferencia, es "revistas electrónicas". La expresión "revistas digitales" sólo es utilizada por 2 bibliotecas.

- Información asociada a los títulos de las revistas: es algo poco frecuente y de escasa variedad. Lo más extendido (en 19 casos, 33\%) es informar sobre el año de inicio; el resto de la información localizada no supera en ningún momento el $16 \%$ de los centros.

La cantidad y variedad de revistas científicas electrónicas disponibles en las bibliotecas universitarias españolas reflejan un reconocimiento y aceptación de esta modalidad de publicación por parte del ámbito académico. Por tanto es un hecho, como afirman Martín y Merlo (2003, p. 155) que la revista electrónica, que nació como complemento a la edición impresa de las publicaciones seriadas, está ganando partida a los soportes tradicionales.

\section{Notas}

1. Según J. Baró i Queralt y J. A. Ontalba Ruipérez, citados por García Gómez, Francisco Javier. "Revistas digitales españolas especializadas en ciencias de la documentación. Autoría y temas de investigación: estudio y análisis". En: La documentación para la investigación: homenaje a José Antonio Martín Fuertes. Vol. II. León: Universidad, Secretariado de Publicaciones y Medios Audiovisuales, 2002, p. 247.

2. Seleccionamos las ventajas recogidas en los siguientes trabajos: García Gómez, Francisco Javier. Op. cit.; Marcos, Mari Carmen. "La revista electrónica y su aceptación en la comunidad científica". En: El profesional de la información, 2000, v. 9, n. 5, pp. 4-14.

3. Por ejemplo los trabajos de Carter, Bordeianu y Dennos (1999), Estivill y Gascón (1999), Beck (2000), Lam (2000), Marcos (2000), Ortoll (2000).

4. Para localizar las webs de las bibliotecas universitarias hemos utilizado la siguiente dirección: Bibliotecas universitarias y de investigación españolas:

http://www.csic.es/cbic/webuni.htm

5. Las tablas se han diseñado mediante filas y columnas, las respuestas afirmativas o negativas se recogen en las celdas correspondientes. En pocas ocasiones no hemos conseguido la información solicitada (bien porque la biblioteca no ha respondido o porque al consultar la web, determinada información o recurso no estaba activado o disponible); esta situación se indica en las celdas mediante el uso de dos guiones (--).
6. La única excepción ha sido la universidad Ramon Llull en la que se diferencia entre Blanquerna, IQS y Esade.

7. El cuestionario ha sido contestado por todas la bibliotecas a excepción de las bibliotecas de la Universidad Autónoma de Madrid y de la Universidad de Deusto. La primera la hemos eliminado del estudio por no poder acceder por internet a ningún dato (es imprescindible la clave de usuario); la segunda la hemos mantenido, pues algunos datos están disponibles a través de la web.

8. Boydston, Jeanne M. K; Leysen, Joan M. "Internet resources cataloguing in ARL libraries: staffing and access issues". En: The serials librarian, 2002, v. 41, n. 3/4, pp. 127-145 y Jordan, Mark; Kisly, Dave.

"How does your library handle electronic serials?: a general survey". En: Serials, 2002, March, v. 15, n. 1, pp. 41-46. Citados por Estivill, Assumpció; Argudo, Sílvia; Miralpeix, Concepció. "Presentació i accés a les revistes electròniques a les universitats catalanes". En: BiD, Biblioteconomia i documentació, desembre, 2002, n. 9. Consultado en: 11-05-04. http://www.ub.es/biblio/bid/09estiv1.htm

\section{Referencias bibliográfic as}

Beck, Melissa. Conser cataloging manual. Module 3, remote access electronic serials (online serials). With the assistance of Bill Anderson, Les Hawkins, and Regina Reynolds. Updated Fall 2000. Library of Congress. Consultado en: 07-06-04.

http://www.loc.gov/acq/conser/module31.html

Carter, Christina E.; Bordeianu, Sever; Dennis, Nancy. "The real world of integrating electronic resources into a web opac". Peter C. Whiting, recorder. En: The serials librarian, 1999, v. 36, n. 3/4, pp. 455-460.

Estivill, Assumpció; Argudo, Sílvia; Miralpeix, Concepció. "Presentació $\mathrm{i}$ accés a les revistes electròniques a les universitats catalanes". En: BiD, Biblioteconomia i documentació, 2002, desembre, n. 9. Consultado en: 11-05-04.

http://www.ub.es/biblio/bid/09estiv1.htm

Estivill, Assumpció; Gascón, Jesús. “Algunos problemas de la catalogación de revistas electrónicas de acceso remoto y el estado actual de revisión de las reglas". En: Item, Revista de biblioteconomia i documentació, 1999, n. 24, pp. 135-152.

García, Consol. "Revistas electrónicas: el acceso y la presentación”. En: VI Jornadas catalanas de documentación, 1997. Barcelona: Socadi, Cobdc, pp. 267-273.

Jordan, Mark; Kisly, Dave. "How does your library handle electronic serials?: a general survey". En: Serials, 2002, March, v. 15, n. 1, pp. $41-46$.

Lam, Vinh-The. "Organizational and technical issues in providing access to electronic journals". En: The serials librarian, 2000, v. 39, n. 3, pp. 25-34.

Marcos, Mari Carmen. "La revista electrónica y su aceptación en la comunidad científica”. En: El profesional de la información, 2000, v. 9, n. 5, pp. 4-14.

Martín González, Juan Carlos; Merlo Vega, José Antonio. "Las revistas electrónicas: características, fuentes de información y medios de acceso". En: Anales de documentación, 2003, n. 6, pp. 155-186.

Olmeda Gómez, Carlos; Catalán Vega, Marcos. "Encuesta sobre publicaciones científicas españolas electrónicas". En: V Congreso ISKO-España, 2001. La representación y organización del conocimiento: metodologías, modelos y aplicaciones. Madrid: Universidad de Alcalá de Henares.

Ortoll Espinet, Eva. “Gestión de acceso a revista electrónicas”. En: Boletín de la Anabad, 2000, v. 50, n. 2, pp. 191-201.

Rich, Linda A.; Rabine, Julie L. "How libraries are providing access to electronic serials: a survey of academic library web sites". En: Serials review, 1999, v. 25, n. 2, pp. 35-46.

José Luis Herrera Morillas, Facultad de Biblioteconomía y Documentación, Universidad de Extremadura, Badajoz. jlhermor@alcazaba.unex.es 